University of New Hampshire

University of New Hampshire Scholars' Repository

Space Science Center

Institute for the Study of Earth, Oceans, and

Space (EOS)

$9-2-2010$

\title{
A portable neutron spectroscope (NSPECT) for detection, imaging and identification of nuclear material
}

James M. Ryan

University of New Hampshire, James.Ryan@unh.edu

Chris Bancroft

University of New Hampshire

Peter F. Bloser

University of New Hampshire, Peter.Bloser@unh.edu

U Bravar

University of New Hampshire - Main Campus

Dominique Fourguette

Michigan Aerospace Corp.

See next page for additional authors

Follow this and additional works at: https://scholars.unh.edu/ssc

Part of the Astrophysics and Astronomy Commons

\section{Recommended Citation}

James M. Ryan ; Christopher Bancroft ; Peter Bloser ; Ulisse Bravar ; Dominique Fourguette ; Colin Frost ; Liane Larocque ; Mark L. McConnell ; Jason Legere ; Jane Pavlich ; Greg Ritter ; Greg Wassick ; Joshua Wood and Richard Woolf "A portable neutron spectroscope (NSPECT) for detection, imaging and identification of nuclear material", Proc. SPIE 7806, Penetrating Radiation Systems and Applications XI, 780607 (September 02, 2010); doi:10.1117/12.860652; http://dx.doi.org/10.1117/12.860652

This Conference Proceeding is brought to you for free and open access by the Institute for the Study of Earth, Oceans, and Space (EOS) at University of New Hampshire Scholars' Repository. It has been accepted for inclusion in Space Science Center by an authorized administrator of University of New Hampshire Scholars' Repository. For more information, please contact Scholarly.Communication@unh.edu. 


\section{Authors}

James M. Ryan, Chris Bancroft, Peter F. Bloser, U Bravar, Dominique Fourguette, Colin Frost, Liane

Larocque, Mark L. McConnell, Jason S. Legere, Jane Pavlich, Greg Ritter, Greg Wassick, Joshua Wood, and R S. Woolf 


\title{
A Portable Neutron Spectroscope (NSPECT) for detection, imaging and identification of nuclear material
}

\author{
James M. Ryan*a, Christopher Bancroft ${ }^{\mathrm{a}}$, Peter Bloser ${ }^{\mathrm{a}}$, Ulisse Bravar ${ }^{\mathrm{a}}$, Dominique Fourguette ${ }^{\mathrm{b}}$, \\ Colin Frost ${ }^{a}$, Liane Larocque ${ }^{b}$, Mark L. McConnell a, Jason Legere ${ }^{\text {a }}$, Jane Pavlich ${ }^{b}$, Greg Ritter ${ }^{\text {, }}$, \\ Greg Wassick, Joshua Wood ${ }^{\text {a, }}$ Richard Woolf ${ }^{\text {a }}$ \\ aSpace Science Center, University of New Hampshire, 8 College Rd., Durham, NH USA 03824; \\ bMichigan Aerospace Corp., 1777 Highland Drive, Suite B, Ann Arbor, MI USA 48108
}

\begin{abstract}
We have developed, fabricated and tested a prototype imaging neutron spectrometer designed for real-time neutron source location and identification. Real-time detection and identification is important for locating materials. These materials, specifically uranium and transuranics, emit neutrons via spontaneous or induced fission. Unlike other forms of radiation (e.g. gamma rays), penetrating neutron emission is very uncommon. The instrument detects these neutrons, constructs images of the emission pattern, and reports the neutron spectrum. The device will be useful for security and proliferation deterrence, as well as for nuclear waste characterization and monitoring. The instrument is optimized for imaging and spectroscopy in the $1-20 \mathrm{MeV}$ range. The detection principle is based upon multiple elastic neutron-proton scatters in organic scintillator. Two detector panel layers are utilized. By measuring the recoil proton and scattered neutron locations and energies, the direction and energy spectrum of the incident neutrons can be determined and discrete and extended sources identified. Event reconstruction yields an image of the source and its location. The hardware is low power, low mass, and rugged. Its modular design allows the user to combine multiple units for increased sensitivity. We will report the results of laboratory testing of the instrument, including exposure to a calibrated Cf-252 source. Instrument parameters include energy and angular resolution, gamma rejection, minimum source identification distances and times, and projected effective area for a fully populated instrument.
\end{abstract}

Keywords: neutrons, imaging, spectroscopy

\section{INTRODUCTION}

There are several applications for a domestic neutron imager/spectrometer, but they all revolve around detecting, locating, monitoring and characterizing nuclear material, often outside a lab environment.

Imaging brings a new aspect to the problems mentioned above. Neutrons are, by their nature, resistant to detection, and defy easy imaging and spectroscopy. Detection has mostly been in the form of registering moderated or thermalized neutrons from a fast neutron source. Because one only measures the charged particles produced by a neutron interaction, deducing the properties of the parent neutron is ambiguous, besides being difficult.

Registering moderated neutrons comes without any information about the incident direction or energy. Measuring fast neutrons in a bulk detector provides a compromised energy measurement, but still lacks directional information. A double-scatter telescope pays the penalties of greatly increased complexity and low efficiency by requiring two neutron scatters, but it benefits in the end because the elastic scatter kinematics can be used to constrain the incident velocity vector while simultaneously performing a quality energy measurement. The $\gamma$-ray analog of this concept is a Compton telescope, a device that has seen extensive use in space for astrophysical research ${ }^{1}$.

\section{THE DOUBLE-SCATTER TECHNIQUE}

Neutrons have no charge, and therefore do not readily interact. Thus, they must be detected by indirect methods. A preferred method for neutron detection at $\mathrm{MeV}$ energies exploits the large elastic n-p scattering cross section. The choice material, serving the functions of neutron scatterer and recoil proton detector, is an organic scintillator. This material consists of mainly hydrogen and carbon, in the number ratio of 1.0 to 2.0. The relative concentration of hydrogen varies with scintillator type.

*james.ryan@unh.edu; phone 1603862 3510; fax 16038623584

Penetrating Radiation Systems and Applications XI, edited by F. Patrick Doty, H. Bradford Barber,

Hans Roehrig, Richard C. Schirato, Proc. of SPIE Vol. 7806, 780607 - (c) 2010 SPIE

CCC code: $0277-786 \mathrm{X} / 10 / \$ 18 \cdot$ doi: $10.1117 / 12.860652$

Proc. of SPIE Vol. $7806780607-1$ 
To perform imaging, an incident neutron must undergo an n-p scatter in each of two detectors. One must be able to follow the path of the neutron once it enters the instrument, measuring the location, relative time and energy deposits of each n-p interaction. Consider the scenario shown in Fig. 1-a neutron, whose incident direction is unknown, as it undergoes two n-p scatters. By measuring the spatial coordinates of the two interactions and time of flight (ToF), one can determine the energy and direction (i.e., momentum vector) of the scattered neutron. By measuring the energy of the first recoil proton, one can then compute the energy of the incident neutron as well as the scatter angle.

With this information one can constrain the incident neutron direction to lie on the mantle of a cone about the recoil neutron velocity vector (Fig. 1). The scatter angle $\sin ^{2} \theta=E_{\mathrm{p}} / E_{\mathrm{n}}$. By projecting the cone onto an object plane or sphere, one has an event circle for each event, also shown in Fig. 1. From the intersection of multiple event circles, through statistical means it is possible to obtain an image of a neutron source. The same basic principle has been employed extensively and successfully for $\gamma$-ray imaging in Compton telescopes to image the complex distribution of point and extended sources in the sky.

\section{DETECTOR DESIGN}

\subsection{Ultimate Detector Concept}

Previous generations of neutron imaging telescopes have been constructed with either plastic or liquid scintillator. Liquid scintillator systems have the advantage of high hydrogen content, relatively good light output, and the ability to perform pulse shape discrimination. Pulse shape discrimination (and ToF) allows the system to reject $\gamma$ rays, important for high background environments.

The instrument design, Fig. 2, is a two layer system using liquid scintillator. The front detector layer is made up of 1" cylindrical cells of liquid scintillator, read out by fast, rugged 1" photomultiplier tubes (PMT). The rear detector layer is similarly comprised of 1" liquid scintillator cells read out by similar PMTs. Time-of-flight measurements are made

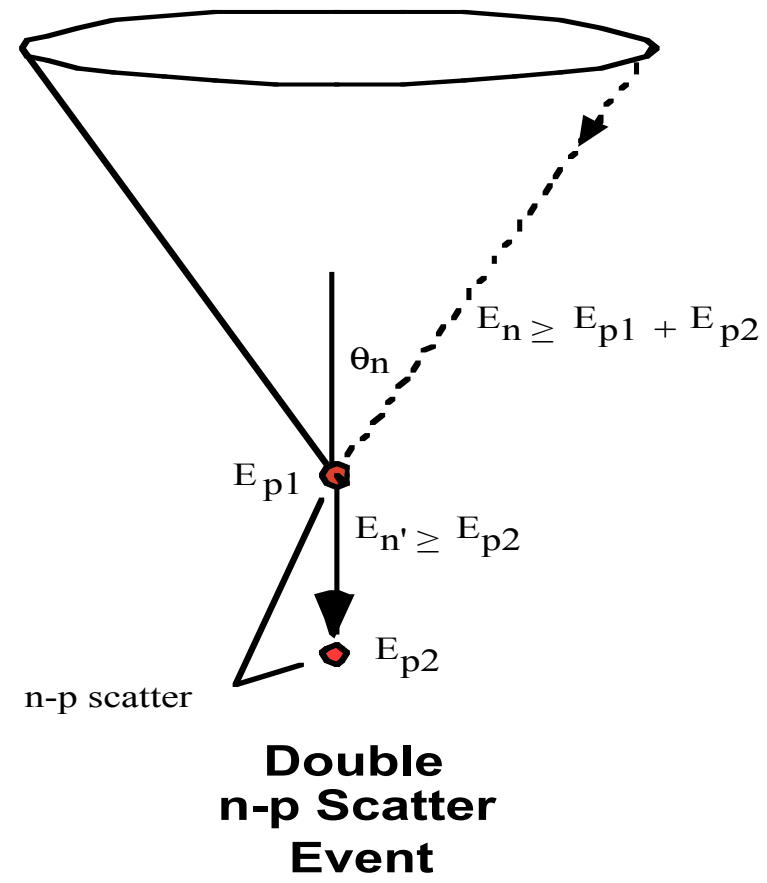

Fig. 1. Neutron double-scatter kinematics.

between planes. All cells represent independent data channels. When a neutron interacts in the forward detector plane D1, a analog signals from that detector plane are generated from the analog sum of the different cells in D1. Those signals initiate the ToF measurement, create a fast logic signal to test for time coincidence with what happens in D2, and serve as an analog pulse height for the energy deposited by the neutron in D1. The cell identification is taken to be the 
interaction location, i.e., a spatial resolution of 1" corresponding to a particular cell. Multiple signals from different D1 cells are rejected. Similar signal processing takes place in D2, where the sum signal stops the ToF measurement and the cells that trigger are identified and recorded.

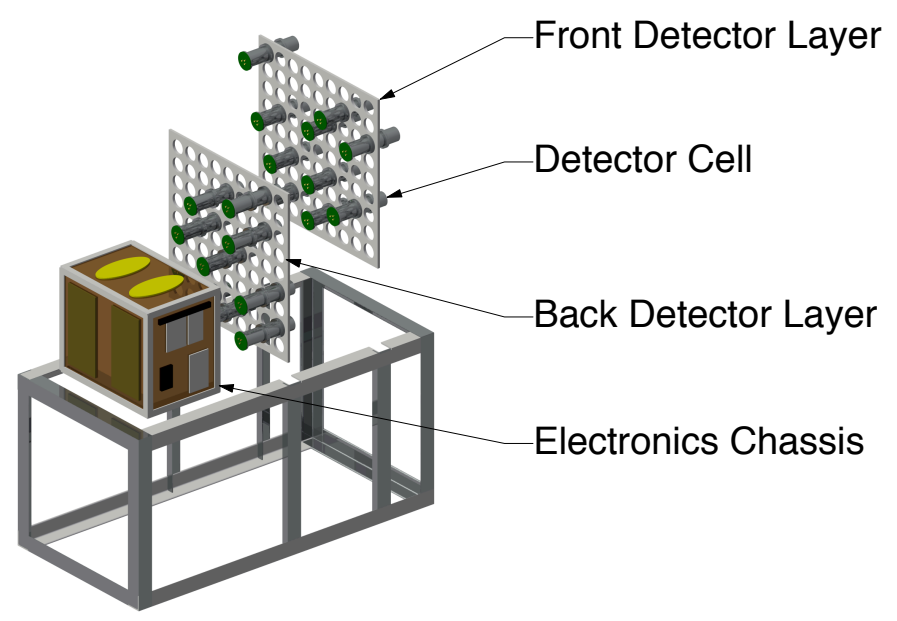

Fig. 2. Design schematic.

An organic scintillator is used that possesses pulse-shape-discrimination properties. This property of the scintillator produces analog signals that can be used to identify whether the ionizing particle is either fast (electron, muon) or slow (proton or heavy ion). The two types of particles differ in their detailed pulse shape - a function of the chemistry of the scintillator. Neutron-initiated events can thus be identified and selected for further data analysis. Electron ionization pulse shapes are generally discarded.

The two planes are separated by $30 \mathrm{~cm}$, scintillator to scintillator. A $\gamma$ ray over this distance registers a $1 \mathrm{~ns}$ ToF, while a $1-\mathrm{MeV}$ neutron requires $\sim 30 \mathrm{~ns}$ to cover the same distance. Oblique trajectories yield longer ToF values, but this effect is corrected with the event location information provided by the cell identifications. The ToF range is of order $50 \mathrm{~ns}$, extending above and below typical fast neutron speeds. By having a longer ToF range, one automatically obtains a measure of the accidental coincidence rate - an important measurement in hot radiation environments. The instrument is designed to operate eight hours on battery power with each plane populated with 77 detector cells. Analog data are processed in an on-board computer, digitized and formatted for transmission to a remote computer for real-time monitoring or analysis and/or data archiving. The connection between the on-board and remote computers can be by ethernet cable or wireless communications.

The instrument is controlled through the remote computer, nominally a laptop computer running LabVIEWTM. The remote computer also monitors many housekeeping parameters, such as temperature, voltages, count rates in all detector cells and other rates, important for assessing instrument behavior and performance. Gains in the PMTs are commandable. The detector cell planes are sprung to absorb shock during transit.

\subsection{Prototype Instrument}

The forward detector plane D1 as of the time of this writing is populated with only three cells. The rear detector is similarly populated with three cells. This is sufficient to test the integration of multiple cells in the two detector planes. The two planes are separated by the proper $30 \mathrm{~cm}$. The cells are screw mounted in a precision aluminum frame to maintain precise registration. The aluminum structure is shock mounted in a chassis that also houses the supporting signal processing electronics, power systems and on-board computer. The control, monitoring and imaging computer is remotely located, and communicates with the on-board computer.

The instrument, at the time of this writing, is in the process of being populated and progressively integrated. It has been tested rigorously at the cell level and the integrated instrument level using a mix of specific instrument electronics or separate rack-mounted electronics in lieu of the final circuitry. 
Lab measurements using a ${ }^{252} \mathrm{Cf}$ source were first performed with single detector cells. The module-pair bench model consists of two liquid scintillator modules separated by $30 \mathrm{~cm}$, conforming to the dimensions of the final instrument. Each detector cell was coupled to a PMT (R1924A) and custom preamplifier; Nuclear Instrumentation Module (NIM) and VME electronics use fast and signals from the preamplifier to measure the time-of-flight (ToF) and pulse shape. Slow signals provide the pulse height (energy deposited) and module ID We irradiated this configuration with a ${ }^{252} \mathrm{Cf}$ fission source at a distance of $3 \mathrm{~m}$, offset by $30^{\circ}$ from the instrument axis.

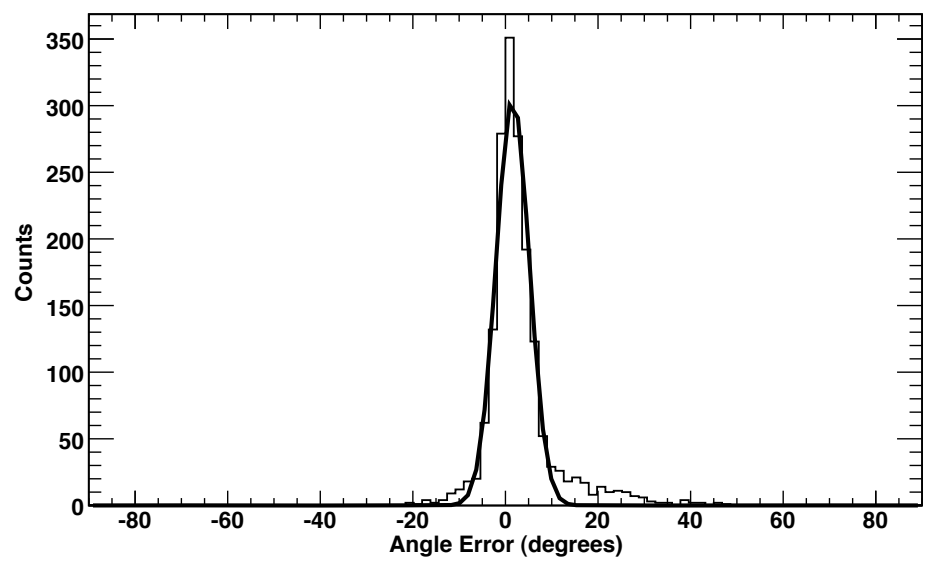

Fig. 3. The ARM distribution of a two-module experiment.

Double scatters were selected using both hardware and software criteria. In hardware, signals from D1 and D2 must occur within $50 \mathrm{~ns}$ of each other to satisfy coincidence requirements and pulse height thresholds were set to $50 \mathrm{keV}_{\text {ee }}$ (electron equivalent). Cuts on ToF and pulse shape were made in software. Established neutron time-of-flight and pulse shape discrimination parameters could be adjusted to expand or contract the data acceptance window for subsequent analysis. Using this information, the angular resolution measurement (ARM) can be measured (Fig, 3). The ARM is the difference between the measured kinematic angle (from energy and ToF) and the geometric scattering angle (knowing the true source position). The width of this distribution is the sum of the uncertainties produced by the spatial, energy and ToF uncertainties and is of order $10^{\circ}$ FWHM (full width half-maximum). The skewness of this distribution represents systematic errors.

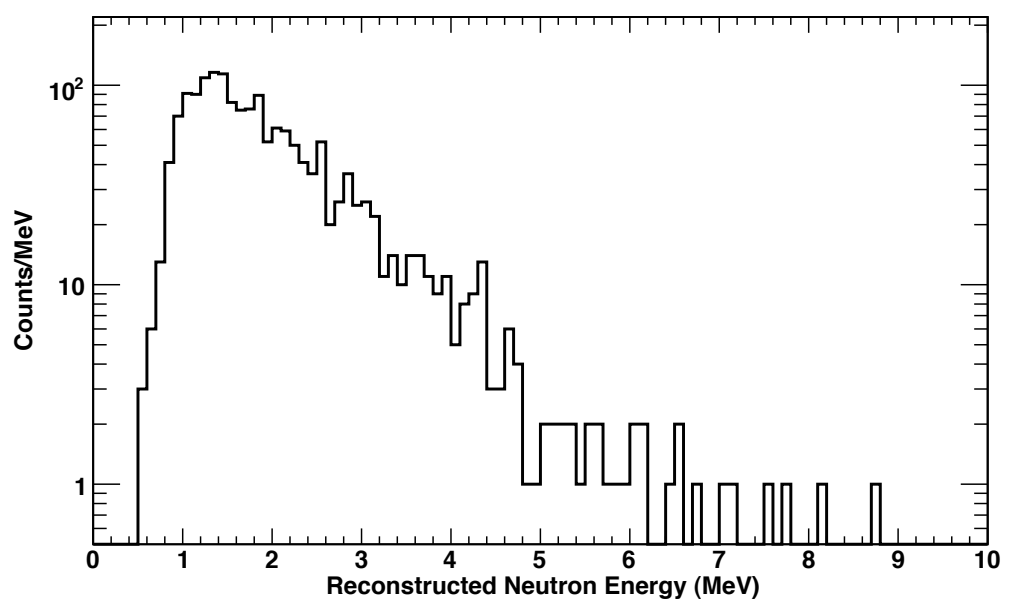

Fig. 4. Neutron count spectrum. 
To select neutrons that are fully measured, we choose events that fall within $\pm 20^{\circ}$ of the mean of the ARM distribution and use them for spectral analysis. These are events that satisfy neutron-proton elastic scattering kinematics over the known trajectory. By construction, the energy measurement must be accurate, broadened only by the instrument resolution with no skewness. The count spectrum was reconstructed on an neutron event-by-event basis; the total neutron energy is the sum of the recoil proton energy deposited in the first detector plus the energy of the scattered neutron determined from ToF. The count spectrum from a long exposure to a ${ }^{252} \mathrm{Cf}$ source is shown in Fig. 4.

The shape of the uncorrected count spectrum (Fig. 4) was fit with an exponential function and follows the form of: $N(E)$ $\approx \exp \left(-0.88^{*} E(\mathrm{MeV})\right)$, consistent with previous measurements ${ }^{3}$. The neutron energy threshold of the D1-D2 prototype is $\sim 300 \mathrm{keV}_{\text {pe }}$. The threshold of the neutron double scatter measurement is driven by the light output of both D1 and D2 and their associated associated front end electronics (FEE). This setup is being optimized with the as-built FEE and processing electronics. The similarity to the Watt spectrum says that the instrument response matrix is both diagonal and relatively flat over the operating energy range.

\section{THE PARTIALLY POPULATED INSTRUMENT}

As mentioned above, the next step after testing two cells in isolation was to use multiple (three) cells for both D1 and D2. This exercise served the following purposes: (1) It tested the performance of the electronics for each plane to simultaneously process signals from multiple cells and (2) it tested our ability to trim the gains and thresholds of six cells, so that they acted like a single instrument with minimal dispersion in the spectrum and image because of gain and threshold variations. The nine cell-pair combinations should behave as one, when each cell is properly adjusted and trimmed. For this exercise, we put the ${ }^{252} \mathrm{Cf}$ source on axis at a distance of $3.4 \mathrm{~m}$.

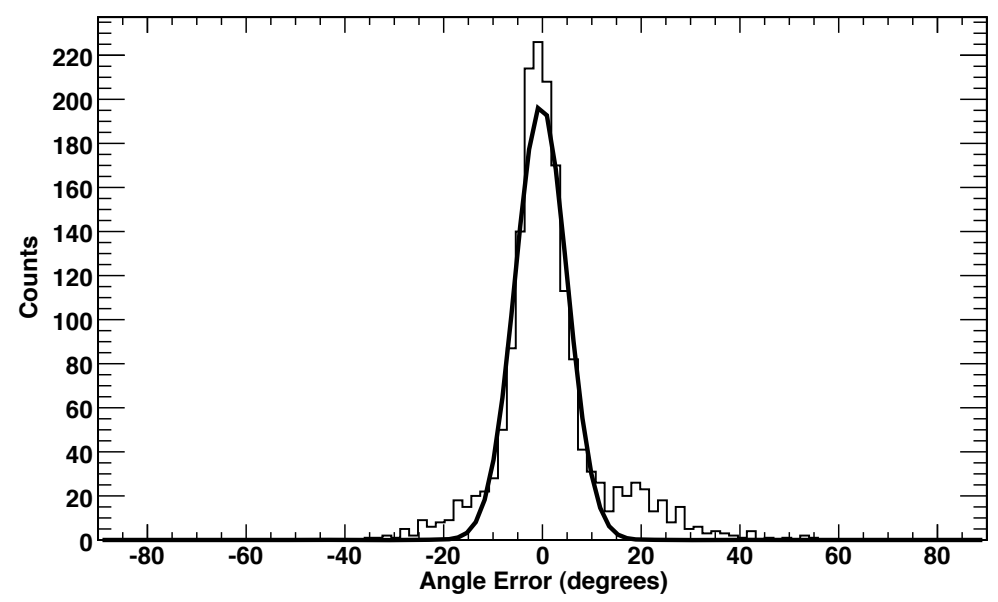

Fig. 5. Data and Gaussian fit to ${ }^{252} \mathrm{Cf}$ neutron source ARM distribution.

Shown in Fig. 5 is the ARM distribution for the $3 \times 3$ cumulative combination. Note that the neutron trajectory for each combination of cells is different and each kinematically measured scattered angle must agree with the scatter angle measured by simple geometry. The ARM function is the difference of those two angles per neutron event. The width of this distribution is $\sim 12^{\circ}$ (FWHM), somewhat larger than that measured with only two cells $\left(10^{\circ} \mathrm{FWHM}\right)$. This is probably due to small variations in the gains of the D1 cells or the ToF calibrations. The non-gaussian wings on the ARM distribution arise from neutron events near the threshold of detection, i.e., $50 \mathrm{keV}_{\mathrm{ee}}$ in either D1 or D2, or equivalently neutrons from approximately $300 \mathrm{keV}$ to $700 \mathrm{keV}$.

To measure the spectrum of the neutron source, we did as before, selecting events for which the measured scatter angle was within $20^{\circ}$ of the true angle. The results, i.e., count spectrum, is shown in Fig. 6. 


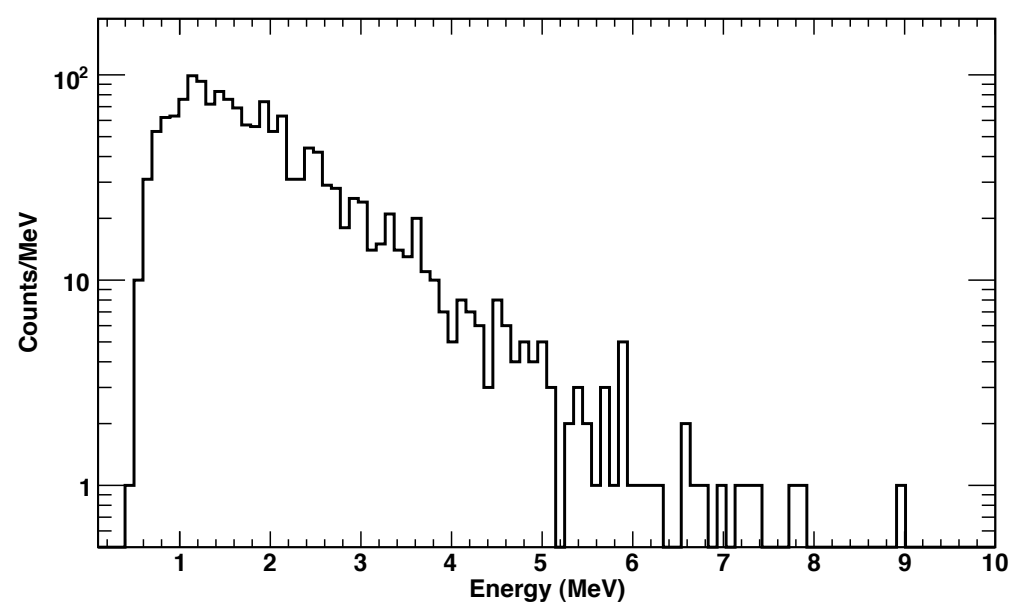

Fig. 6. The count spectrum from the partially populated prototype.

The important feature to note here, but not readily seen, is the far greater number of counts per unit live time compared to the two-cell experiment. This is due to the $9 \times$ larger effective area compared to the two-cell configuration. The exponential Watt shape is still recovered and is smoother due to the greater statistics.

Lastly, the imaging capability can now be examined with many (nine) different cell geometries with correspondingly different event cone axes, allowing event circles to intersect. The 3-d plot of the circle intersection density is shown in Fig. 7. No ARM cuts were applied to construct this plot. ARM cuts are only applied to construct the energy spectrum once the source position is known.

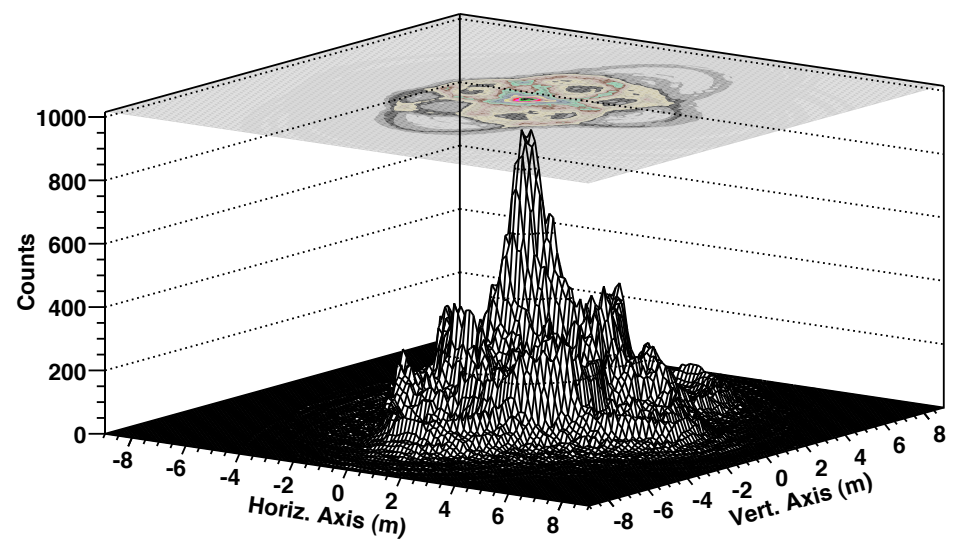

Fig. 7. A 3-d plot of event circle intersection density, i.e., image, of the point source for the partially populated prototype.

A strong central peak in Fig. 7 is present in the image plane corresponding to true location of the source. However, there is considerable structure outside the central peak. This structure arises from the correlated circle intersections from the presence of a point source. They are most apparent when the number of cell pairs is small, i.e., nine in our case. With a fully populated instrument, the cell pair count grows to 100 and these lobes or ghost images will disperse, while the central peak grows in proportion to the effective area, i.e., proportional to the number of cell pairs. 


\section{CONCLUSIONS}

NSPECT, the neutron spectrometer, under fabrication and integration by Michigan Aerospace Corp. and the University of New Hampshire has been exercised as an operating imaging spectrometer. Resolution figures for energy and angle are comparable to those taken under ideal conditions for two cells. However, nine cell combinations act as one to produce a source image with $9 \times$ the effective area of two operating cells.

By early fall 2010, the instrument will be populated with ten cells per plane, yielding $100 \times$ the effective area of two cells, while producing images with less granularity and greater definition. At this point, advanced testing will be conducted in and outside the laboratory.

\section{ACKNOWLEDGEMENTS}

This work is supported by the Defense Threat Reduction Agency through Michigan Aerospace Corp., contract HDTRA1-08-C-0077. It builds on research started with funding from NNSA NA-22.

\section{REFERENCES}

[1] Schönfelder, V. et al., "Instrument Description and Performance of the Imaging Gamma-Ray Telescope COMPTEL Aboard the Compton Gamma-Ray Observatory,” Astrophys. Jour. Supp. Ser. 86, 657-692 (1993).

[2] Bravar, U., Woolf, R.S., Bruillard, P.J., Flückiger, E.O., Legere, J.S., MacKinnon, A.L., Macri, J.R., Mallik, P.C., McConnell, M.L., Pirard, B., and Ryan, J.M., "Calibration of the Fast Neutron Imaging Telescope (FNIT) Prototype Detector,” IEEE Trans. Nuc. Sci. 56, 2947-2954 (2009). 